\title{
Abundancia y diversidad de Bacillus thuringiensis de diferentes hábitat en tres municipios de Boyacá, Colombia
}

\section{Abundance and diversity of Bacillus thuringiensis in different habitats within three municipalities of Boyaca, Colombia}

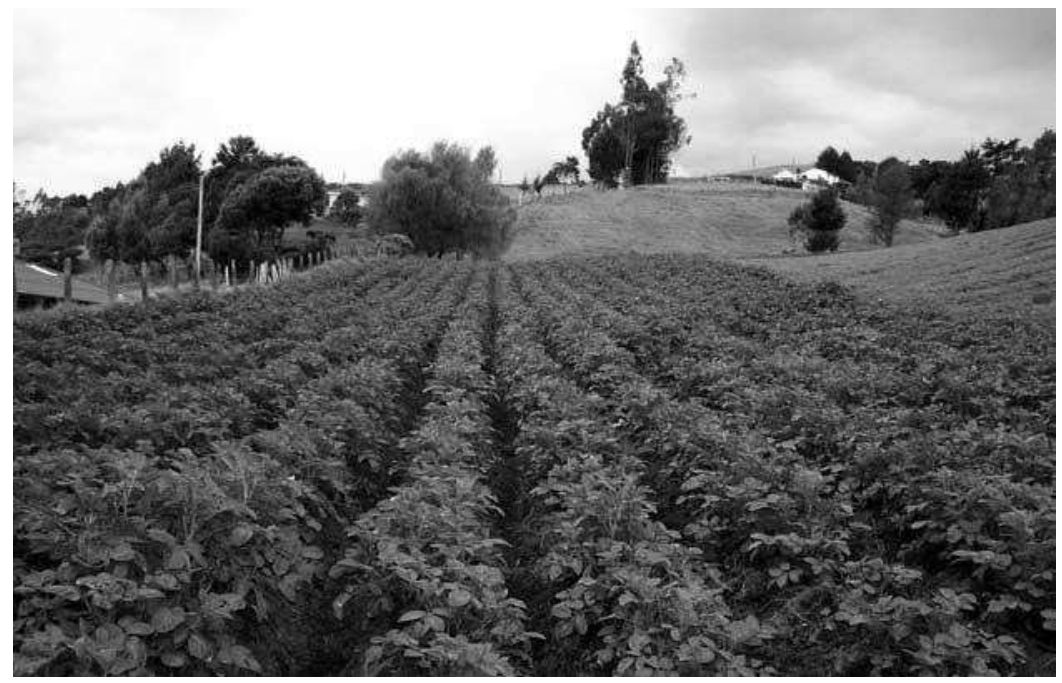

Plantación de papa en el municipio de Tunja, vereda Puente de Boyacá.

Foto: J.M. Poveda.

\section{RESUMEN}

La actual orientación de la agricultura hacia la sostenibilidad y los estudios recientes sobre el impacto social y económico que ha causado el abuso de plaguicidas químicos ha generado un creciente interés por promover desarrollos en el campo de los bioplaguicidas que contribuyan a la solución de problemas empleando estrategias acordes con las condiciones de cada región. Bacillus thuringiensis $(B t)$ es un microorganismo cosmopolita que se constituye en una herramienta valiosa para el control biológico de plagas. Asimismo, es reconocido entre los plaguicidas microbianos de mayor importancia comercial debido a su toxicidad específica contra insectos blanco e inocuidad para el hombre y los animales. El objetivo de este trabajo fue comparar la distribución, abundancia y diversidad de $B t$ provenientes de diferentes hábitats existentes en tres municipios de la provincia Centro de Boyacá (Chíquiza, Siachoque y Tuta), con el fin de iniciar una colección de este microorganismo en el laboratorio de Control Biológico de la UPTC. De 102 aislamientos se obtuvieron 51 cepas de $B t$ de las cuales el $78 \%$ provenía de ecosistemas agrícolas. Del total de bacterias viables de $B t$ en los bosques fueron obtenidas $0,25-2,9010^{5} \mathrm{ufc} / \mathrm{mL}$ y $0,09-2,9810^{5} \mathrm{ufc} / \mathrm{mL}$ en cultivos de maíz, con índices de diversidad de 1,00. Los resultados mostraron la presencia de $B t$ en todos los municipios, aun en aquellos sitios perturbados constantemente por las labores agrícolas.

1 Grupo Manejo Biológico de Cultivos, Facultad de Ciencias Agropecuarias, Universidad Pedagógica y Tecnológica de Colombia (UPTC), Tunja (Colombia).

2 Autor para correspondencia. john.martinez@uptc.edu.co 


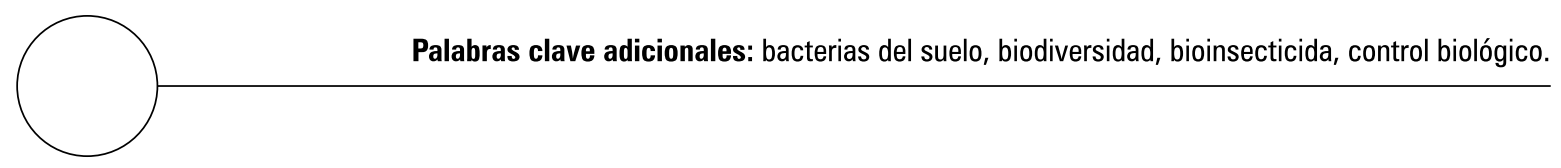

\section{ABSTRACT}

The current orientation of agriculture toward sustainable development, and studies related to economic and social impacts of chemical pesticide abuse have increased interest in biopesticide technology. This has led to the creation of novel solutions specific the phytosanitary problems of each region. Bacillus thuringiensis (Bt) is a cosmopolitan microorganism that has provided the most valuable biological pest control tool worldwide, due to its specificity and low risk to humans and animals. The objective of the present work was to compare distribution, abundance and diversity of $B t$ from different habitats in three localities (Chiquiza, Siachoque $y$ Tuta) of the central province of Boyaca, Colombia, with the goal of initiating a Bt collection in the Laboratory of Biological Control of UPTC. Starting from a 102 isolates, 51 Bt strains were obtained, out of which 78\% originated from agricultural systems. Viable Bt bacteria were obtained from forests $(0.25$ to $2.90105 \mathrm{cfu} / \mathrm{mL})$, and maize crops ( 0.09 to $2.98105 \mathrm{cfu} / \mathrm{mL}$ ), with diversity indexes of 1.00 . These results indicated that the $B t$ is present in the three localities studied, even in sites usually disturbed by agricultural activities.

Additional keywords: soil bacteria, biodiversity, bioinsecticides, biological control.

Fecha de recepción: 10-03-2008

Aprobado para publicación: 03-06-2008

\section{INTRODUCCIÓN}

Boyacá es un departamento netamente agrícola con diversidad de suelos que surte de alimentos a una buena parte de la región central y norte de Colombia. Uno de los factores que más dificulta la producción agrícola en el departamento es el manejo de los problemas fitosanitarios, causados por enfermedades y plagas, cuyo control representa un alto porcentaje en la estructura de costos del cultivo. Existe en las zonas una arraigada tradición de uso de agroquímicos, los cuales, además de ser ineficientes en muchos casos, generan resistencia en los insectos plaga, afectan al medio ambiente, contaminan las cosechas y, en muchos casos, tienen efectos directos en la salud de los productores o consumidores.

En un mercado internacional, en el que la competencia y las restricciones frente a la presencia de residuos químicos adquieren gran importancia, la agricultura colombiana y boyacense debe promover el uso de alternativas de producción limpia que partan de nuestra diversidad, permitan la producción sostenible de los cultivos, garanticen la protección del medio ambiente, favorezcan la calidad y con un beneficio común. Actualmente, se conocen estrategias biológicas de manejo de plagas desarrolladadas a partir de la exploración de la diversidad microbiana de diferentes regiones.

Bacillus thuringiensis ( $B t)$ es una bacteria entomopatógena del suelo, aeróbica facultativa, gram positiva, caracterizada por la producción de un cuerpo paraesporal o cristal de proteínas conocidas como delta-endotoxinas o proteínas Cry. Estos cristales se forman durante el proceso de es- 
porulación y en ellas reside la actividad tóxica de $B t$ hacia larvas de diferentes órdenes de insectos, cuando son ingeridas (Hofte y Whiteley, 1989; Feitelson et al., 1992; Cerón et al., 1995; Ibarra et al., 2003; Tamez-G. et al., 2005). Dichos cristales presentan diferentes formas: bipiramidales, esféricas, cuadradas, entre otras (Lynch y Baumann, 1985; Höfte y Whiteley, 1989; Rodríguez et al., 1991). Se ha demostrado que las inclusiones de esta bacteria contienen, principalmente, proteínas de 25 a $140 \mathrm{KDa}$ (Restrepo et al., 1997).

Dadas las características de $B t$ como agente de control biológico y debido a su presencia cosmopolita, sus esporas se han aislado de diversas fuentes como suelo (Martin y Travers, 1989; Hossain et al., 1997; Bravo et al., 1998), agua (Iriarte et al., 2000), hojas de plantas (Wlodzimierz y Elzbieta, 1999), insectos muertos (Chilcot y Wigley, 1993; Kaelin et al., 1994; Apoloyo et al., 1995; Kim et al., 1998), productos almacenados (Meadows et al., 1992; Kaelin et al., 1994; Xavier et al., 2007) y telarañas. Los bioinsecticidas derivados de esta bacteria se han utilizado por más de 40 años (Ibarra y López, 2000).

El uso de productos a base de $B t$ se ha generalizado principalmente en cultivos de hortalizas, cereales y algodón, representando más del $90 \%$ de los bioplaguicidas que se comercializan en el mundo (Yara et al., 1999; Vallejo y Orduz, 1996; Bravo y Cerón, 2004; Adams et al., 1996), constituyéndose en el insecticida de origen biológico de mayor importancia y eficiencia en el control biológico de diversas plagas agrícolas en el mundo (Feitelson et al., 1992); todo esto ha conducido al establecimiento de colecciones que suman cerca de 40.000 aislados, concentradas en grandes firmas privadas que controlan el mercado mundial (Lambert y Peferoen, 1992).

Algunos de los factores que han hecho posible su éxito en la agricultura, además de su aprobación por la Organización Mundial de la Salud, son su alta biodegradabilidad, alta especificidad
(Deacon, 1983) e inocuidad para mamíferos, otros vertebrados, plantas e inclusive otros insectos benéficos (Shelton et al., 2002; Ibarra et al., 2003), siendo una alternativa promisoria y ambientalmente segura en el control de insectos plaga nocivos.

Por otro lado, la aparición de resistencia a algunas toxinas de Bt (Restrepo et al., 1998) y el control ineficiente de los insecticidas químicos (Pérez et al., 1997), indica que la utilización masiva de bioinsecticidas a base de $B t$ debe estar apoyada por productos que puedan competir, en precio y disponibilidad, con los insecticidas químicos (Vallejo y Orduz, 1996); esto ha llevado tanto a las industrias como a los laboratorios y centros de investigación a enfocar sus esfuerzos en la búsqueda de nuevas moléculas, especialmente de bacterias entomopatógenas, para el desarrollo de alternativas novedosas de control (Bravo et al., 1998; Silva et al., 1999), lo cual ha contribuido a ampliar el conocimiento de la distribución ecológica de este microorganismo en la naturaleza (Uribe et al., 2003). Actualmente, los volúmenes de uso de esta bacteria están limitados al número de insectos susceptibles a los productos comerciales existentes, necesitándose aislar y probar nuevas cepas y aislamientos.

En Colombia, algunos grupos de investigación han caracterizado cepas nativas de $B t$, enfocando el trabajo hacia lepidópteros como Spodopetra frugiperda, Heliothis virescens (Cerón et al., 1995) y T. solanivora (Martínez et al., 2002) y dípteros implicados en problemas de salud pública como Aedes aegyptii y Culex quinquefasciatus (Díaz et al., 1993). Cada vez más se reconoce el papel e importancia de la biodiversidad en el funcionamiento de los sistemas agrícolas; sin embargo, hasta el momento, trabajos sistemáticos sobre caracterización de la diversidad de cepa nativas de Bt en Boyacá no se han desarrollado, limitando la generación de nuevos registros de $B t$ para Boyacá y Colombia que puedan llegar a ser de importancia económica regional o nacional. 
El objetivo de este trabajo fue comparar la distribución, abundancia y diversidad de $B t$ provenientes de diferentes hábitats existentes en tres municipios de la provincia Centro de Boyacá

\section{MATERIALES Y MÉTODOS}

Recolección de muestras. Un total de 32 muestras de suelo fueron recolectadas en bolsas plás- ticas estériles, en 12 diferentes hábitas existentes en zonas de bosques naturales, pantanos y zonas agrícolas de los municipios de Chiquiza, Siachoque, y Tuta de la Provincia, Centro del departamento de Boyacá (tabla 1). Las muestras se recolectaron eliminando totalmente la vegetación que cubría el suelo en cada sitio, tomando para cada muestra, con una cuchara diferente, $250 \mathrm{~g}$ de suelo, hasta una profundidad de $7 \mathrm{~cm}$. Las muestras se llevaron al laboratorio, donde se

Tabla 1. Abundancia y diversidad de aislamientos de B. thuringiensis en Boyacá, Colombia.

\begin{tabular}{|c|c|c|c|c|c|c|}
\hline Origen & $\begin{array}{l}\text { Muestra } \\
\text { de suelo }\end{array}$ & $\begin{array}{l}\text { No. de bacterias } \\
\times 10^{6} / \mathrm{mL} \text { antes de } \\
\text { la pasteurización }\end{array}$ & $\begin{array}{l}\text { No. de } \\
\text { esporas formadas } \\
\times 10^{5} / \mathrm{mL}\end{array}$ & $\begin{array}{l}\text { No. de } B \text {. } \\
\text { thuringiensis } \\
\times 10^{5} / \mathrm{mL}\end{array}$ & $\begin{array}{l}\text { Porcentaje } \\
\text { de } \boldsymbol{B} \text {. } \\
\text { thuringiensis }\end{array}$ & $\begin{array}{l}\text { Índice de } \\
\text { diversidad }{ }^{1}\end{array}$ \\
\hline \multirow{6}{*}{$\begin{array}{l}\text { Bosques } \\
\text { naturales }\end{array}$} & 1 & 0,31 & $2,87(9,3)$ & 2,90 & 9,3 & $1,00(5 / 5)$ \\
\hline & 2 & 0,69 & $1,61(2,4)$ & 0,25 & 0,4 & $0,50(1 / 2)$ \\
\hline & 3 & 0,20 & $3,01(15,1)$ & 1,29 & 6,5 & $0,29(2 / 7)$ \\
\hline & 4 & 0,17 & $0,71(4,3)$ & 0,00 & 0,0 & $0,00(0 / 0)$ \\
\hline & 5 & 0,33 & $1,22(8,9)$ & 0,00 & 0,0 & $0,00(0 / 1)$ \\
\hline & 6 & 0,40 & $0,48(1,2)$ & 0,00 & 0,0 & $0,00(0 / 0)$ \\
\hline Pantanos & 7 & 0,48 & $1,32(2,8)$ & 1,31 & 2,8 & $1,00(3 / 3)$ \\
\hline \multirow{4}{*}{ Maíz } & 8 & 0,18 & $2,91(16,1)$ & 2,91 & 16,1 & $1,00(8 / 8)$ \\
\hline & 9 & 0,25 & $2,08(8,5)$ & 1,05 & 4,3 & $0,67(2 / 3)$ \\
\hline & 10 & 0,17 & $2,97(18,0)$ & 2,98 & 18,0 & $1,00(2 / 2)$ \\
\hline & 11 & 0,65 & $2,76(4,2)$ & 0,09 & 0,1 & $022(2 / 9)$ \\
\hline Trigo & 12 & 2,86 & $1,14(0,4)$ & 0,00 & 0,0 & $0,00(0 / 1)$ \\
\hline \multirow{2}{*}{ Avena } & 13 & 2,89 & $0,67(0,2)$ & 0,00 & 0,0 & $0,00(0 / 1)$ \\
\hline & 14 & 0,34 & $1,20(3,6)$ & 0,16 & 0,5 & $0,43(3 / 7)$ \\
\hline \multirow{3}{*}{ Leguminosas } & 15 & 0,25 & $2,98(11,9)$ & 0,00 & 0,0 & $0,00(0 / 3)$ \\
\hline & 16 & 0,82 & $2,74(3,3)$ & 0,00 & 0,0 & $0,00(0 / 1)$ \\
\hline & 17 & 1,26 & $0,63(0,5)$ & 0,63 & 0,5 & $1,00(2 / 2)$ \\
\hline \multirow{5}{*}{ Papa } & 18 & 0,26 & $2,16(8,3)$ & 0,05 & 0,2 & $0,50(1 / 2)$ \\
\hline & 19 & 0,30 & $0,15(0,5)$ & 0,59 & 2,0 & $0,67(2 / 3)$ \\
\hline & 20 & 0,83 & $0,44(0,5)$ & 0,16 & 0,2 & $0,50(2 / 4)$ \\
\hline & 21 & 0,11 & $0,05(0,4)$ & 0,00 & 0,0 & $0,00(0 / 0)$ \\
\hline & 22 & 1,21 & $0,46(0,4)$ & 0,46 & 0,4 & $1,00(2 / 2)$ \\
\hline \multirow{2}{*}{ Pastos } & 23 & 0,28 & $0,76(2,8)$ & 0,23 & 0,8 & $0,33(1 / 3)$ \\
\hline & 24 & 0,40 & $2,41(6,0)$ & 0,06 & 0,2 & $0,33(2 / 6)$ \\
\hline \multirow{3}{*}{ Rábano } & 25 & 2,88 & $1,25(0,4)$ & 0,00 & 0,0 & $0,00(0 / 1)$ \\
\hline & 26 & 0,61 & $1,17(1,9)$ & 0,18 & 0,3 & $0,50(1 / 2)$ \\
\hline & 27 & 0,82 & $1,80(2,2)$ & 0,30 & 0,4 & $0,50(1 / 2)$ \\
\hline \multirow{2}{*}{$\begin{array}{l}\text { Cebolla } \\
\text { cabezona }\end{array}$} & 28 & 1,31 & $1,04(0,8)$ & 0,49 & 0,4 & $0,501 / 2)$ \\
\hline & 29 & 1,19 & $2,70(2,3)$ & 0,00 & 0,0 & $0,00(0 / 1)$ \\
\hline \multirow{2}{*}{ Frutales } & 30 & 0,56 & $2,07(3,7)$ & 0,22 & 0,4 & $0,40(2 / 5)$ \\
\hline & 31 & 0,33 & $1,43(4,3)$ & 0,40 & 1,2 & $0,50(4 / 8)$ \\
\hline Calabaza & 32 & 1,31 & $0,36(0,3)$ & 1,09 & 0,8 & $0,33(2 / 6)$ \\
\hline
\end{tabular}

1 Índice de diversidad $(X Y)$ es la diversidad de colonias de $B$. thuringiensis $(X)$ sobre la diversidad de colonias de Bacillus sp. (Y). 
secaron a temperatura ambiente; posteriormente, se homogenizaron con rodillo y se tamizaron en tamices de 850 y 500 micrones. Las muestras procesadas se almacenaron a una temperatura de $4^{\circ} \mathrm{C}$, hasta su respectivo análisis. En un formato individual y para cada muestra, se recopilaron datos como: vereda, finca, propietario, tipo de cultivo, estado de desarrollo, labores culturales y riego.

Aislamiento de bacterias. El aislamiento de $B a-$ cillus thuringiensis y otras formas esporales de bacterias se hizo de acuerdo con el método descrito por Travers et al. (1987). En agua destilada estéril se suspendió un gramo de cada muestra de suelo y se pasteurizó a $80^{\circ} \mathrm{C}$ por media hora, seguidamente se realizaron diluciones y se sembraron en agar nutritivo e incubaron a $30^{\circ} \mathrm{C}$ durante $24 \mathrm{~h}$. Para la selección de cepas de Bacillus thuringiensis, se suspendieron $0,5 \mathrm{~g}$ de cada muestra de suelo procesada, en matraz de $20 \mathrm{~mL}$, con $10 \mathrm{~mL}$ de caldo Luria Bertani (LB) y Acetato de Na 0,25M (Travers et al., 1987) previamente esterilizados. Posteriormente, se llevó este material a agitación continua a $200 \mathrm{rpm}$, durante $12 \mathrm{~h}$ a $29^{\circ} \mathrm{C}$. Pasado este tiempo, las muestras se calentaron a $80^{\circ} \mathrm{C}$ durante 3 min en baño maría. De esta suspensión, se realizaron diluciones desde $10^{-1}$ hasta $10^{-4}$ con agua destilada estéril, las cuales fueron sembradas por duplicado en medio LB $\left(10 \mathrm{~g} \mathrm{~L}^{-1}\right.$ triptona, $5 \mathrm{~g} \mathrm{~L}^{-1}$ extracto de levadura, $10 \mathrm{~g} \mathrm{~L}^{-1}$ $\mathrm{NaCl}$ y $15 \mathrm{~g} \mathrm{~L}^{-1}$ de agar) e incubadas a $30^{\circ} \mathrm{C}$ por $48 \mathrm{~h}$. El recuento de bacterias viables esporuladas se realizó después de la pasteurización y el recuento de colonias de $B$. thuringiensis después de llevar a cabo el método de selección por acetato.

Identificación de Bacillus thuringiensis. Se determinaron las colonias características de la bacteria, realizando tinciones simples con cristal violeta $(0,5 \%)$ y verde de malaquita, detectando por microscopía a 100X células vegetativas, esporas o cristales (Porcar y Juárez, 2004). Finalmente, las colonias típicas de $B t$ se repicaron en medio LB y se incubaron nuevamente a $30^{\circ} \mathrm{C}$, hasta alcanzar esporulación, para verificar la presencia del cuerpo paraesporal y definir su forma.
Conservación de cepas. Las cepas de Bacillus thuringiensis identificadas y caracterizadas se conservaron en tiras de papel filtro, siguiendo la metodología descrita por Porcar y Juárez (2004), en viales ámbar de $20 \mathrm{~mL}$ y sellados con agrafes. El material se almacenó en cajas de cartón para protección contra la luz y permanecen en el Laboratorio de Control Biológico de la UPTC en Tunja, Colombia.

\section{RESULTADOS Y DISCUSIÓN}

Se determinó la presencia de cepas nativas de $B$. thuringiensis, obtenidas en 32 muestras de suelo, recolectadas en 12 hábitats diferentes de los municipios de Chíquiza, Siachoque y Tuta, situados en la Provincia Centro del departamento de Boyacá, municipios seleccionados por su diversidad de agroecosistemas. El aislamiento de las cepas nativas de $B t$ se hizo en seis muestras de suelo provenientes de zonas protegidas (Bosques $\mathrm{Na}$ turales), pantanos (una muestra) y 25 muestras provenientes de áreas agrícolas (tabla 1). El mayor recuento de bacterias viables antes de la pasteurización $\left(\sim 2,8010^{6} \mathrm{ufc} / \mathrm{mL}\right)$ se encontró en áreas cultivadas con trigo, avena y rábano, mientras que en el cultivo de papa se determinó el menor valor de bacterias viables $\left(0,1110^{6} \mathrm{ufc} / \mathrm{mL}\right)$.

Los resultados evidencian que las áreas de bosques presentan el contenido más alto de bacterias esporuladas $\left(0,48-3,0110^{5} \mathrm{ufc} / \mathrm{mL}\right)$ con un $1,2-15,1 \%$, seguido por los suelos cultivados con maíz $(2,08$ $2,9710^{5} \mathrm{ufc} / \mathrm{mL}$ ) con $4,2 \%$ y $18,0 \%$ (tabla 1 ), En cuanto a $B$. thuringiensis, se observaron $0,25-2,90$ $10^{5} \mathrm{ufc} / \mathrm{mL}$, para bosques, y $0,09-2,9810^{5} \mathrm{ufc} / \mathrm{mL}$ para maíz con un $0,4-9,3 \%$ y $0,1-18,0 \%$, del total de bacterias viables, respectivamente. Estos rangos son inferiores comparados con los reportados por Chatterjee et al. (2007) en tierras de la India con rangos entre 4,23-6,52 $10^{5} \mathrm{ufc} / \mathrm{mL}$.

En zonas protegidas y áreas cultivadas con maíz, papa y frutales, se encontró la mayor abundancia de esporas y bacterias de B. thuringiensis, mientras que en zonas cultivadas con trigo no se 
encontraron cepas productoras del cuerpo paraesporal (tabla 2).

En la tabla 1 se evidencia que las cepas de B. thuringiensis predominaron entre las cepas de Bacillus sp. con índice de diversidad de 1,00 para las muestras $1,7,8,10,17$ y 22 pertenecientes a suelos de bosques, pantanos, maíz, leguminosas y papa, información que se asemeja a la reportada por Phyllis et al. (1989). Los índices de diversidad de las demás muestras oscilan entre 0,0 y 0,67, datos similares a los índices reportados para suelos de España que oscilan entre 0,067 y 0,11 (Quesada-Moraga et al., 2004). La mayor diversidad de colonias del genero Bacillus se encontró en suelos cultivados con maíz nueve colonias (tabla 1), seguida por suelos cultivados con frutales ocho colonias y suelos cultivados con avena y áreas de bosques naturales siete colonias; la más alta diversidad de colonias de $B$. thuringiensis se encontró en áreas de maíz, ocho colonias, segui- das por cinco colonias en áreas de bosques, frutales, cuatro colonias, y pantanos, tres colonias; se obtuvieron pocas colonias de $B t$ de los suelos de leguminosas, papa, rábano y cebolla cabezona (tabla 1). Se obtuvieron 51 cepas de Bt, de las cuales el $78 \%$ provenían de ecosistemas agrícolas, destacándose las áreas cultivadas con maíz con $27 \%$, similar a Valicente y Barrero (2003), que aislaron la mayoría de cepas de $B t$ en zonas productoras de maíz en Brasil. El 22\% restante correspondió a hábitats de bosques naturales y pantanos; es importante mencionar que tanto el $50 \%$ de las muestras de suelo, pertenecientes a bosques naturales, como el $72 \%$ de las muestras, pertenecientes a zonas agrícolas, presentaron al menos una cepa de Bt. Por otro lado, en el ecosistema de pantanos el 100\% registró presencia de Bt (tabla 2).

La mayor presencia de Bt en ecosistemas agrícolas coincide con lo reportado por Xavier et al. (2007);

Tabla 2. Distribución de Bacillus thuringiensis aislados de las muestras de suelos boyacenses, colombianos.

\begin{tabular}{|c|c|c|c|c|c|}
\hline \multirow[b]{2}{*}{ Origen } & \multicolumn{2}{|c|}{ No. de muestras de suelo } & \multicolumn{2}{|c|}{ No. de colonias } & \multirow{2}{*}{$\begin{array}{c}\text { pH } \\
\text { del suelo }\end{array}$} \\
\hline & Recolectadas & Con Bt $(\%)$ & Examinadas & $\begin{array}{c}B t \\
(\% \text { del total })\end{array}$ & \\
\hline Bosques naturales & 6 & $3(50)$ & 15 & $8(16)$ & 4,20 \\
\hline Pantanos & 1 & $1(100)$ & 3 & $3(6)$ & 5,09 \\
\hline Maíz & 4 & $4(100)$ & 22 & $14(27)$ & 5,82 \\
\hline Avena & 2 & $1(50)$ & 8 & $3(6)$ & 4,60 \\
\hline Leguminosas & 3 & $1(33)$ & 6 & $2(4)$ & 4,67 \\
\hline Рapa & 5 & $4(80)$ & 11 & $7(14)$ & 3,94 \\
\hline Pastos & 2 & $2(100)$ & 9 & $3(6)$ & 4,06 \\
\hline Rábano & 3 & $2(67)$ & 5 & $2(4)$ & 4,32 \\
\hline Cebolla cabezona & 2 & $1(50)$ & 3 & $1(2)$ & 4,31 \\
\hline Frutales & 2 & $2(100)$ & 13 & $6(12)$ & 6,04 \\
\hline Calabaza & 1 & $1(100)$ & 6 & $2(4)$ & 5,16 \\
\hline Trigo & 1 & $0(0)$ & 1 & $0(0)$ & 4,22 \\
\hline Subtotal & 25 & $18(72)$ & 84 & $40(78)$ & \\
\hline Total & 32 & $22(69)$ & 102 & $51(100)$ & \\
\hline
\end{tabular}


en relación con las zonas protegidas evaluadas, los datos concuerdan con lo descrito por Martín y Travers (1989), quienes obtuvieron un $87,1 \%$ de $B t$ en suelos agrícolas y $66,7 \%$ para muestras de bosques de lluvia tropical; Sin embargo, otros trabajos como los de Chilcot y Wigley (1993) y Yara et al. (1999), evidenciaron mayores porcentajes de $B t$ en muestras de suelo recolectadas de bosques nativos, con al menos una cepa de $B t$ presente, en comparación con los suelos agrícolas cuyos porcentajes fueron inferiores.

Trabajos más recientes como los de Iriarte et al. (2000) e Itsimatsu et al. (2000), afirman que las muestras provenientes de zonas pantanosas o con baja intensidad de corriente pueden contener B. thuringiensis en una frecuencia relativamente alta, que concuerda con este estudio, en el que de una sola muestra de pantano se identificaron tres cepas diferentes, correspondientes al $6 \%$ del total de las cepas obtenidas. Las muestras de suelo de zonas pantanosas son una buena fuente de esporas bacterianas, debido a que las esporas se sedimentan rápidamente, conservando su viabilidad al estar protegidas por los rayos solares, además son refugios favorables para algunas especies de insectos, lo que permite la presencia de cepas de $B t$ en este medio (Mohammedi et al., 2006).

La ubicuidad de $B t$ encontrado en una amplia variedad de hábitats (Bravo et al., 1998) ha sido demostrada en diversos trabajos, ya que tiene la capacidad para soportar en estado latente (esporas) condiciones adversas del medio ambiente, lo que facilita su larga supervivencia (Sneath, 1986).

En este estudio en las muestras de suelo recolectadas, se determinó un pH ácido (tabla 2), con mayoría de texturas franco-arcillosas y francolimosas, similares a las texturas de las que se ha obtenido gran diversidad de cepas de Bt en otros cultivos como arroz (Chatterjee et al., 2007); sin embargo, Bacillus thuringiensis fue detectado en los distintos tipos de suelos muestreados independientemente de su ecofisiografía, lo cual indica que su ocurrencia no muestra ninguna relación directa con el pH del suelo. Esta característica química interactuaría con otros factores importantes, como son nutrientes, humedad, cantidad de materia orgánica, que garantizan el éxito en la supervivencia de una población de $B t$, a pesar de su pobre capacidad para crecer plenamente en una comunidad edáfica.

Cuando se realizó el análisis por región de origen (tabla 3), se observó que los municipios de Siachoque y Chíquiza cuentan con la más alta diversidad de especies tanto de Bacillus sp. como de $B t$, con 19 y 17 colonias, correspondiente al 37\% y $33 \%$ del total de cepas de Bt obtenidas. Aunque en el municipio de Chíquiza se obtuvó el mayor número de muestras de suelo analizadas, sólo presentó un $44 \%$ de éstas, con presencia de $B t$, a diferencia de Siachoque, donde hubo presencia de al menos una cepa de $B t$ en todas las muestras de suelo recolectadas.

Tabla 3. Distribución de Bacillus thuringiensis en muestras de suelo, por región de origen.

\begin{tabular}{|c|c|c|c|c|c|}
\hline \multirow[b]{2}{*}{ Municipios } & \multicolumn{2}{|c|}{ No. de muestras de suelo } & \multicolumn{2}{|c|}{ No. de colonias } & \multirow{2}{*}{$\begin{array}{l}\text { Porcentaje del } \\
\text { total de cepas de } \\
\text { Bt obtenidas }\end{array}$} \\
\hline & Recolectadas & $\begin{array}{c}\text { Con al menos una } \\
\text { cepa de } B t(\%)\end{array}$ & $\begin{array}{l}\text { Seleccionadas } \\
\text { diferentes a } B t\end{array}$ & $B t(\%)$ & \\
\hline Chíquiza & 16 & $7(44)$ & 33 & $17(52)$ & 33 \\
\hline Tuta & 6 & $5(83)$ & 24 & $15(63)$ & 29 \\
\hline Siachoque & 10 & $10(100)$ & 45 & $19(42)$ & 37 \\
\hline Total & 32 & $22(69)$ & 102 & $51(50)$ & 100 \\
\hline
\end{tabular}


Esta menor presencia puede estar relacionada con la existencia de una agricultura intensiva de monocultivos, con gran uso de productos químicos para mantener su producción y controlar plagas, lo que, probablemente afecta de manera negativa la diversidad microbiológica de sus suelos.

Por otro lado, en el municipio de Tuta se aisló el $63 \%$ de las colonias de Bacillus thuringiensis; sin embargo, este municipio presenta el menor índice de diversidad comparado con los otros municipios.

La selección de cepas de $B t$, por sus características macroscópicas y microscópicas; reveló la presencia de una inclusión o cristales en los esporangios (células bacilares con una espora en su interior), siendo estos de forma bipiramidal, triangular, redonda, cuadrada y amorfa (tabla 4). La gran mayoría de los aislamientos con estos tipos de cristales corresponden a muestras de cultivos de cereales obtenidos de zonas agrícolas y otras de áreas protegidas (tabla 1).
Es importante señalar que la forma bipiramidal de los cristales fácilmente observable (figura 1), predomina en suelos de bosque natural y suelos cultivados con maíz y papa, lo cual concuerda con los últimos estudios realizados en varias regiones de Colombia por Armengol et al. (2007), donde éste es el tipo de cristal más frecuente, con el $60 \%$ de abundancia; igualmente, la forma bipiramidal es muy común al ser aislada de comida para animales (Meadows et al., 1992). Un alto porcentaje de aislamientos presentaron inclusiones paraesporales de forma triangular-redonda no muy frecuentes en la morfología típica de $B$. thuringiensis en cuanto al cristal se refiere; la gran mayoría de estos aislamientos corresponde a zonas de bosques, pantanos, cultivos de avena, leguminosas, pastos y calabaza. En general, los cristales cuadrados fueron menos frecuentes, sin embargo el tipo cuadrado-redondo se observó en aislamientos obtenidos de suelos cultivados con frutales y calabaza, con $67 \%$ y $50 \%$ del total, respectivamente (tabla 4 ).

Tabla 4. Distribución de los tipos morfológicos de cuerpos paraesporales por lugar de origen.

\begin{tabular}{|c|c|c|c|c|c|c|c|c|c|}
\hline \multirow{2}{*}{ Origen } & \multicolumn{8}{|c|}{ Número de cepas (\% del tipo de cristal) } & \multirow{2}{*}{$\begin{array}{l}\text { Total de } \\
\text { cepas }\end{array}$} \\
\hline & B & $A-0$ & $\mathrm{~A}-\mathrm{T}$ & $\mathrm{C}-\mathrm{T}$ & $\mathrm{C}-0$ & $\mathrm{~T}-0$ & T-B-C & $\mathrm{T}-\mathrm{C}-\mathrm{O}$ & \\
\hline Bosque natural & $3(38)$ & $1(13)$ & - & $1(13)$ & - & $3(38)$ & - & - & $8(100)$ \\
\hline Pantanos & $1(33)$ & - & - & - & - & $1(33)$ & $1(33)$ & - & $3(100)$ \\
\hline Maíz & $6(43)$ & $3(21)$ & $1(7)$ & $1(7)$ & $2(14)$ & $1(7)$ & - & - & $14(100)$ \\
\hline Avena & - & - & - & $1(33)$ & $1(33)$ & $1(33)$ & - & - & $3(100)$ \\
\hline Leguminosas & - & $1(50)$ & - & - & - & $1(50)$ & - & - & $2(100)$ \\
\hline Рapa & $3(43)$ & - & $1(14)$ & $1(14)$ & - & $1(14)$ & - & $1(14)$ & $7(100)$ \\
\hline Pastos & $1(33)$ & - & - & - & $1(33)$ & $1(33)$ & - & - & $3(100)$ \\
\hline Rábano & - & - & - & $1(50)$ & - & - & $1(50)$ & - & $2(100)$ \\
\hline Cebolla cabezona & - & - & - & $1(100)$ & - & - & - & - & $1(100)$ \\
\hline Frutales & - & $1(17)$ & - & - & $4(67)$ & $1(17)$ & - & - & $6(100)$ \\
\hline Calabaza & - & - & - & - & $1(50)$ & $1(50)$ & - & - & $2(100)$ \\
\hline Total & $14(27)$ & $6(12)$ & $2(4)$ & $6(12)$ & $9(18)$ & $11(22)$ & $2(4)$ & $1(2)$ & $51(100)$ \\
\hline
\end{tabular}

Clasificación Tipos Morfológicos de inclusiones paraesporales.

B, Bipiramidal; A-0 Amorfo-Redondo; A-T amorfo-triangular; C-T cuadrado-triangular; $\mathrm{C}-0$ cuadrado-redondo; T-0 triangular-redondo; T-B-C triangular-bipiramidal y cuadrado o rectangular; $\mathrm{T}-\mathrm{C}-0$ triangular-cuadrado-redondo. 
En un aislamiento obtenido del municipio de Siachoque, especialmente de cultivos de papa, se observaron inclusiones paraesporales de tipo triangular, cuadrado y redondo (figura 2), característica poco común y escasamente registrados en la literatura (Yara et al., 1999). Encontrar cepas con características poco comunes en suelos Boyacenses muestra la gran diversidad de Bt. De esta forma, nuestros resultados contribuyen al conocimiento de la diversidad de Bt en Colombia, donde existen muy pocas colecciones caracterizadas, en comparación con el resto de América latina (Bravo et al., 1998).

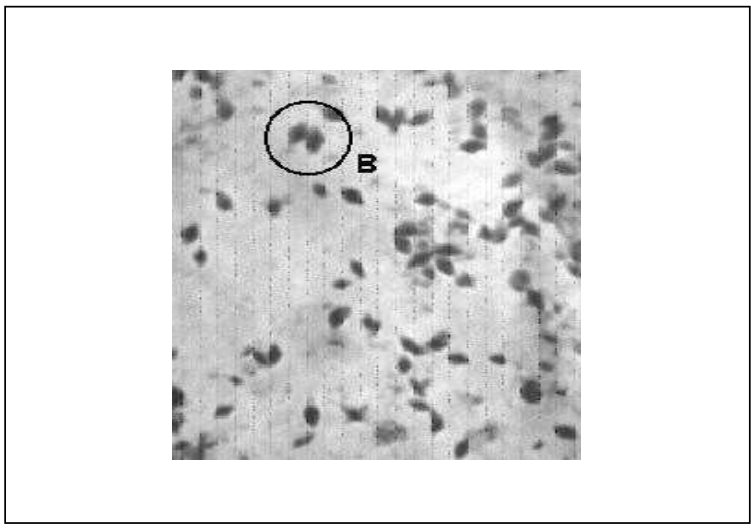

Figura 1. Visualización microscópica de una cepa nativa de Bacillus thuringiensis proveniente de cultivos de maíz. Cristales de forma bipiramidal (B).

\section{CONCLUSIONES}

Este estudio mostró que el $69 \%$ de las muestras de suelo analizadas en la Provincia Centro del departamento de Boyacá contiene al menos una cepa de $B t$, valor considerable, teniendo en

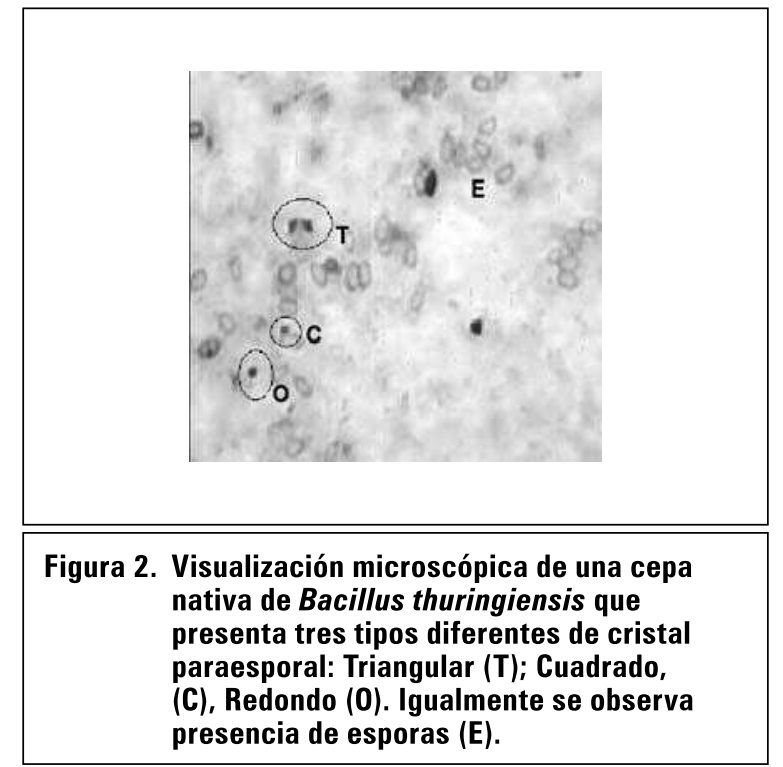

cuenta que estudios en diferentes ecosistemas de Colombia han reportado porcentajes del $82 \%$ (Uribe et al., 2003).

Este estudio es un aporte importante al inicio de un censo de la diversidad de Bt en Boyacá, con el fin de contar con nuevos genes y proteínas activas para el desarrollo futuro de bioplaguicidas para el control de plagas de importancia agrícola de la región.

Aunque en los ecosistemas agrícolas como maíz, frutales, pastos, papa y calabaza se determinó diversidad, los bosques naturales y pantanos fueron una buena fuente de diversidad microbiana, que debe seguir siendo protegida y explorada.

Esta investigación constituyó la base para la creación de una colección de referencia, a partir de la obtención y caracterización de aislamientos de $B t$ de suelos.

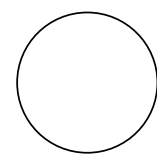

AGRADECIMIENTOS

Este proyecto fue desarrollado con el apoyo de Colciencias y la DIN (UPTC Tunja), por medio del programa de jóvenes investigadores. Los autores agradecen al Dr. Jairo Cerón y la Dra. María Victoria Grosso del Grupo de Biopesticidas del Instituto de Biotecnología de la Universidad Nacional de Colombia, Bogotá, por sus invaluables aportes para la realización de este trabajo. 


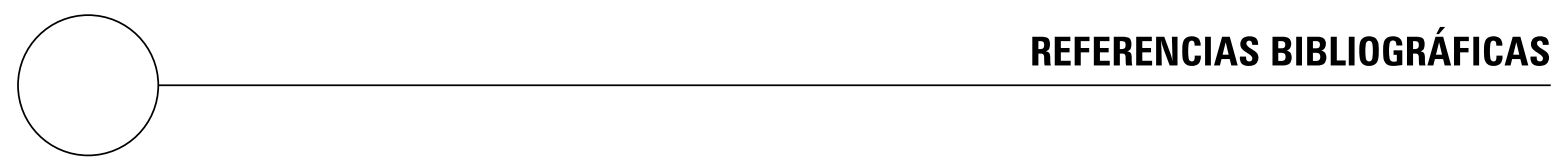

Adams, I.F.; C.I. Liu; S. Macintosh y R.L. Stamen. 1996. Diversity and biological activity of Bacillus thuringiensis. pp. 360-386. En: Coppin, E.L. (ed.). Crop protection agents from nature. Natural products and analogues. The Royal Society of Chemistry, Cambridge, UK.

Apoloyo, C.I.; L. Drif; J.M. Vassal; H. Debarjac; J.P. Bossy; F. Leclant y R. Frutos. 1995. Isolation of múltiple subspecies of Bacillus thuringiensis from a population of the European sunflower moth, Homoeosoma nebulella. Appl. Environ. Microbiol. 61(2), 4243-4347.

Armengol, G.; M.C. Escobar; M.E. Maldonado y S. Orduz. 2007. Diversity of Colombian strains of Bacillus thuringiensis against dipteran and lepidopteran insects. J. Appl. Microbiol. 1(62), 77-88.

Bravo, A.; S. Sarabia; L. Lopez; E. Ontiveros; C. Abarca; A. Ortiz; M. Ortiz; L. Lina; F. Villalobos; G. Peña; M.A. Núñez; M. Soberon y R. Quintero. 1998. Characterization of cry genes in a Mexican Bacillus thuringiensis strain Collection. Appl. Environ. Microbiol. 64, 4965-4972.

Bravo, A. y J. Cerón (eds.). 2004. Bacillus thuringiensis en el control biológico. Universidad Nacional de Colombia, Ed. Buena Semilla, Bogotá.

Cerón, J.; A. Ortiz; R. Quintero; L. Queresa y A. Bravo. 1995. Specific PCR primers directed to indentify Cry I and Cry III genes within a Bacillus thuringiensis strain collection. Appl. Environ. Microbiol. 61(1), 3826-3831.

Chatterjee, S.N.; T. Bhattacharya; T.K. Dangar y G. Chandra. 2007. Ecology and diversity of Bacillus thuringiensis in soil environment. Afric. J. Biotech. 13(6), 1587-1591.

Chilcot, C.N. y P.J. Wigley. 1993. Isolation and toxicity of Bacillus thuringiensis from soil and insect habitats in New Zeland. J. Invert. Pathol. 61, 244-247.

Deacon, J.W. 1983. Microbial control of plant pests and diseases. American Society for Microbiology, Washington, DC.

Díaz, T.; N. Restrepo; S. Orduz y W. Rojas. 1993. Distribución y aislamiento de Bacillus thuringiensis en Colombia. Rev. Coloma. Entomol. 19(2), 35-40.

Feitelson, J.S.; J. Payne y L. Kim. 1992. Bacillus thuringiensis, Insects and Beyond. BioTechnol. 4(10), 271-276.
Höfte, H. y H.R. Whiteley. 1989. Insecticidal crystal proteins of Bacillus thuringiensis. Microbiol. Rev. 53, 242-255.

Hossain, M.A.; S. Ahmed y S. Hoque. 1997. Abundance and distribution of Bacillus thuringiensis in the agricultural soil of Banglasdesh. J. Invert. Pathol. 70, 221-225.

Ibarra, J.E. y M. López. 2000. Bacterias entomopatógenas. Cap. 23. pp. 281-295. En: Badii, M.; A.E. Flores y L.J. Galán Wong (eds.). Fundamentos y perspectivas de control biológico. Universidad Autónoma de Nuevo León, Monterrey, México.

Ibarra, J.E.; M. Soberón y A. Bravo. 2003. La biotecnología y el control biológico de insectos. pp. 27-52. En: Bolívar Z., F.G. y L. Herrera E. (eds.). Fronteras de la biología en los inicios del siglo XXI. Módulo 3: Biotecnología agrícola. El Colegio Nacional, México D.F.

Iriarte, J.; M. Porcar.; M. Lecadet y P. Caballero. 2000. Isolation and characterization of Bacillus thuringiensis strains from aquatic environments in Spain. Curr. Microbiol. 40, 402-408.

Itsimatsu, T.; E. Mizuki; K. Nishimura; T. Akao; H. Sayito; K. Higuchi y M. Ohba. 2000. Occurrence of Bacillus thuringiensis in fresh waters of Japan. Curr. Microbiol. 40, 217-220.

Kaelin, P.; M. Morel y F. Gadani. 1994. Isolation of Bacillus thuringiensis from stored tabaco and Lasioderma serricorne (F.). Appl. Environ. Microbiol. 60(1), 1925.

Kim, H.S.; D.W. Lee; S.D. Woo; Y.M. Yu y S.K. Kang. 1998. Seasonal distribution and characterization of Bacillus thuringiensis isolated from sericultural environments in Korea. J. Gen. Appl. Microbiol. 44, 133-138.

Lambert, B. y M. Peferoen. 1992. Insecticidal promise of Bacillus thuringiensis. Facts and mysteries about a successful biopesticide. Bio Science 2(42), 112-121.

Lynch, M.J. y P. Baumann. 1985. Immunological comparisons of the crystal protein from strains of $\mathrm{Ba}$ cillus thuringiensis. J. Invert. Pathol. 46, 47-57.

Martin, P. y R. Travers. 1989. Worldwide abundance and distribution of Bacillus thuringiensis isolates. Appl. Environ. Microbiol. 55(10), 2437-2442. 
Martínez, W.; D. Uribe y J. Cerón. 2002. Efecto tóxico de proteínas Cry1 de $B$. thuringiensis en larvas Tecia solanivora Povolny. p. 65. En: Memorias Primer Congreso Colombiano de Biotecnología. 26-28 de Junio 2002. Bogotá.

Meadows, M.P.; D.J. Ellis; J. Butt; P. Jarret y H.D. Burges. 1992. Distribution, frequency, and diversity of Bacillus thuringiensis an animal feed mill. Appl. Environ. Microbiol. 58, 1344-1350.

Mohammedi, S.; S.S. Bala; S. Yan; R.D. Tyagi y J.R. Valero. 2006. Molecular screening of Bacillus thuringiensis strains from wastewater sludge for biopesticide production. Proc. Biochem. 41(4), 829-835.

Pérez, Y.O.; A. Rodríguez y A.M. Cotes. 1997. Método rápido y confiable para evaluar la actividad insecticida de cepas nativas de Bacillus thuringiensis contra Tecia solanivora (Polvony) (Lepidóptera: Gelechiidae). Rev. Entomol. Colomb. 3-4(23), 113-118.

Phyllis, A.; W. Martin y R.S. Travers. 1989. Worldwide abundance and distribution of Bacillus thuringiensis isolates. Appl. Environ. Microbiol. 10(55), 2437-2442.

Porcar, M.M. y V. Juarez. 2004. Aislamiento y establecimiento de una colección de Bacillus thuringiensis. Capítulo 6. pp. 151-176. En: Bravo, A. y J. Cerón. (eds.). Bacillus thuringiensis en el control biológico. Universidad Nacional de Colombia, Ed. Buena Semilla, Bogotá.

Quesada-Moraga, E.; E. García-Tovar; P. Valverde-García y C. Santiago-Álvarez. 2004. Isolation, geographical diversity and insecticidal activity of Bacillus thuringiensis from soils in Spain. Microbiol. Res. 1(159), 59-71.

Restrepo, H.N.; D. Gutirerrez.; M.M. Patiño; I. Thiéry; A. Delécluse y S. Orduz. 1997. Clonaje, Expresión y Toxicidad de un gen tóxico de Bacillus thuringiensis subsp. Rev. Colomb. Entomol. 23, 97-101.

Restrepo, H.N.; M. Patiño; S. Orduz; T. Diaz y W. Rojas. 1998. Caracterización de los genes de las toxinas de Bacillus thuringiensis ssp. Rev. Colomb. Entomol. 24, 7-12.

Rodríguez, M.; M.M. De la Torre y N.E. Urquijo. 1991. Bacillus thuringiensis: características biológicas y perspectivas de producción. Rev. Lat. Amer. Microbiol. 33, 279-292.
Shelton, A.M.; J.Z. Zhao y R.T. Roush. 2002. Economic, ecological, food safety, and social consequences of the deployment of $B t$ transgenic plants. Annu. Rev. Entomol. 47, 845-881.

Silva, J.; M. De-Souza; S.J. Díaz y R. Bergmann. 1999. Characterization of Bacillus thuringiensis Subsp. Kurstaki strain S93 effective against the fall armyworm (Spodoptera frugiperda). Canad. J. Microbiol. 45, 464-471.

Sneath, P.H.A. 1986. Endospore-forming gram-positive rods and cocci. pp. 1104-1207. En: Butler, J.P. (ed). Bergey's manual of systematic entomology. Vol 2. Williams and Wilkins, Baltimore, MD.

Tamez-G., P.; M. Iracheta; A.B. Pereyra; W.L. Galán; F.R. Gómez; G. Támez y P. Rodríguez. 2005. Caracterización de cepas mexicanas de Bacillus thuringiensis tóxicas para larvas de lepidópteros y coleópteros. Ciencia UANL 8(4), 447-482.

Travers, R.S.; P.A. Martin y C.F. Reicheldelfer. 1987. Selective process for efficient isolation of soil Bacillus spp. Appl. Environ. Microbiol. 53(6), 1263-1266.

Uribe, D.; W. Martínez y J. Cerón. 2003. Distribution and diversity of cry genes in native strains of Bacillus thuringiensis obtained from different ecosystems from Colombia. J. Invert. Pathol. 82(2), 119-127.

Valicente F.H. y M.R. Barreto. 2003. Bacillus thuringiensis survey in Brazil: geographical distribution and insecticidal activity against Spodoptera frugiperda (J.E. Smith) (Lepidoptera: Noctuidae). Neotrop. Entomol. 32(4), 639-644.

Vallejo, L.F. y P.S. Orduz. 1996. Producción de un plaguicida a base de Bacillus thuringiensis subsp. Kurstaki, a nivel de laboratorio. Rev. Colomb. Entomol. 22, 61-67.

Wlodzimierz, D. y L. Elzbieta. 1999. Biodiversity of Bacillus thuringiensis strains in the phylloplane and soil of Lower Silesia region (Poland). Acta Microbiol. Polon. 48(4), 355-361.

Xavier, R.; C.M. Reena y S. Sreeramanan. 2007. Environmental distribution and diversity of insecticidal proteinas of Bacillus thuringiensis Berliner. Malays. J. Microbiol. 3(2), 1-6.

Yara, E.; J. Cerón y D. Uribe. 1999. Caracterización de aislamientos nativos de Bacillus thuringiensis provenientes de bosque natural y zonas de cultivos colombianos. Rev. Colomb. Entomol. 25, 185-190. 